\title{
Measurable entanglement
}

Alexander Klyachko, Barış Öztop, and Alexander S. Shumovsky

Citation: Appl. Phys. Lett. 88, 124102 (2006); doi: 10.1063/1.2187398

View online: http://dx.doi.org/10.1063/1.2187398

View Table of Contents: http://aip.scitation.org/toc/apl/88/12

Published by the American Institute of Physics

\section{Articles you may be interested in}

Entanglement guides quantum computation

AIP Conference Proceedings 864, 114 (2006); 10.1063/1.2400883

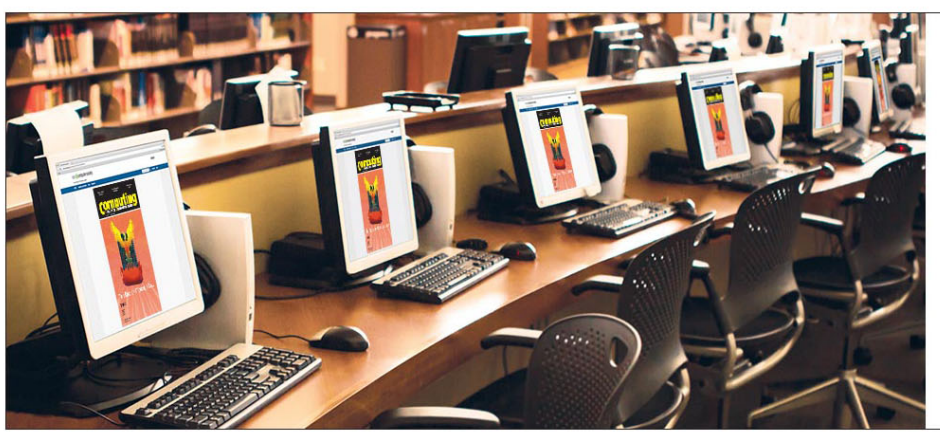

CiSE is already at your fingertips... 


\title{
Measurable entanglement
}

\author{
Alexander Klyachko, Barış Öztop, ${ }^{\text {a) }}$ and Alexander S. Shumovsky \\ Faculty of Science, Bilkent University, Bilkent, Ankara 06800, Turkey
}

(Received 22 December 2005; accepted 2 February 2006; published online 21 March 2006)

The amount of entanglement carried by a quantum bipartite state is usually evaluated in terms of concurrence [S. Hill and W. K. Wootters, Phys. Rev. Lett. 78, 5022 (1997); P. Rungta, V. Bužek, C. M. Caves, M. Hillery, and G. J. Milburn, Phys. Rev. A 64, 042315 (2001)]. We give a physical interpretation of concurrence that reveals a way of its direct measurement and discuss possible generalizations. (C) 2006 American Institute of Physics. [DOI: 10.1063/1.2187398]

Entanglement, which has been considered for decades in the context of fundamentals of quantum mechanics, turns now more and more into a key tool of practical realization of quantum information technologies. The quantum key distribution $^{1}$ for completely secured communications should be mentioned here, first of all (e.g., see Ref. 2).

The design and manufacturing of generators of entangled states require a control of amount of entanglement carried by the states. For pure state $\psi$ of bipartite systems $\mathcal{H}_{A} \otimes \mathcal{H}_{B}$ of format $n \times n\left(n=\operatorname{dim} \mathcal{H}_{A, B} ; n=2\right.$ corresponds to qubits, $n=3$ corresponds to qutrits, etc.), this quantity is given by the concurrence

$$
C(\psi)=\sqrt{v\left[1-\operatorname{Tr}\left(\rho_{r}^{2}\right)\right]},
$$

that has been proposed in Ref. 3 . Here, $\rho_{r}$ denotes the reduced (single-party) density matrix, corresponding to the state $\psi$, and we use the normalization factor $v=n / n-1$, to reduce the concurrence to the interval $[0,1]$. See Ref. 4 for further discussion.

The aim of this letter is to give a natural physical interpretation of the concurrence, and thus to show a way of direct measurement of the amount of bipartite entanglement in terms of mean values of certain physical quantities. Our approach also suggests a general definition of the concurrence for multipartite systems discussed below.

It has been shown in Ref. 5 that entanglement, like coherence and squeezing, can be associated with quantum fluctuations or quantum uncertainties, which are minimal for coherent (separable) states and maximal for completely entangled states. The fluctuations are measured by the total variance defined by the equation

$$
V(\psi)=\sum_{\alpha}\left\langle\psi\left|X_{\alpha}^{2}\right| \psi\right\rangle-\left\langle\psi\left|X_{\alpha}\right| \psi\right\rangle^{2}
$$

where the sum is extended over orthonormal basis $X_{\alpha}$ of Lie algebra of local observables. The basic observables $X_{\alpha}$ act on one of the components: $X_{\alpha}=X_{i}^{A}$ or $X_{\alpha}=X_{j}^{B}$, where $X_{i}^{A}$ and $X_{j}^{B}$ are are orthonormal bases in the space of traceless Hermitian operators in $\mathcal{H}_{A}$ and $\mathcal{H}_{B}$, respectively. It is important to realize that the total variance is independent of the choice of the basic observables $X_{\alpha}$.

The total uncertainty of all basic observables attains its maximal value in the case of completely entangled states (like Bell states of two qubits).

\footnotetext{
${ }^{a)}$ Electronic mail: boztop@fen.bilkent.edu.tr
}

The first sum in the right-hand side of Eq. (2) is independent of the state $\psi$. In fact, the sum

$$
C=\sum_{\alpha} X_{\alpha}^{2}
$$

known as Casimir operator, ${ }^{6,7}$ acts as a multiplication by a scalar $C_{A B}$ (equal to 6 for two qubits, for example). Thus,

$$
V(\psi)=C_{A B}-\sum_{i}\left\langle\psi\left|X_{i}\right| \psi\right\rangle^{2},
$$

so that the measurement of the total uncertainty is reduced to the measurement of mean values of basic observables in the right-hand side of Eq. (3). In the case of complete entanglement,

$$
\left\langle\psi\left|X_{\alpha}\right| \psi\right\rangle=0
$$

for all $\alpha$ (see Ref. 5), so that the total uncertainty achieves its maximum.

We now show that concurrence (1) can be equivalently expressed in terms of the total uncertainty (3) in the case of bipartite systems. Consider first the case of two qubits with the state

$$
|\psi\rangle=\sum_{\ell, \ell^{\prime}=0}^{1} \psi_{\ell \ell^{\prime}}\left|\ell, \ell^{\prime}\right\rangle, \quad \sum_{\ell, \ell^{\prime}=0}^{1}\left|\psi_{\ell \ell^{\prime}}\right|^{2}=1,
$$

where $\left|\ell, \ell^{\prime}\right\rangle \equiv|\ell\rangle \otimes\left|\ell^{\prime}\right\rangle$ denotes a composite state. It can be easily seen that the concurrence (1) is then cast to the form

$$
\begin{aligned}
& C(\psi)=2\left|\psi_{00} \psi_{11}-\psi_{01} \psi_{10}\right|, \\
& =2\left[\left|\psi_{00}\right|^{2}\left|\psi_{11}\right|^{2}+\left|\psi_{01}\right|^{2}\left|\psi_{10}\right|^{2},-2 \operatorname{Re}\left(\psi_{00} \psi_{11} \psi_{01}^{*} \psi_{10}^{*}\right)\right]^{1 / 2} .
\end{aligned}
$$

On the other hand, using Pauli operators,

$$
\begin{aligned}
& \sigma_{x}=|0\rangle\langle 1|+| 1\rangle\langle 0|, \\
& \sigma_{y}=-i(|0\rangle\langle 1|-| 1\rangle\langle 0|), \\
& \sigma_{z}=|0\rangle\langle 0|-| 1\rangle\langle 1|
\end{aligned}
$$

as the basic local observables $X_{i}^{A}$ and $X_{j}^{B}$, one gets

$$
\begin{aligned}
V(\psi)= & 4+4\left[\left|\psi_{00}\right|^{2}\left|\psi_{11}\right|^{2}+\left|\psi_{01}\right|^{2}\left|\psi_{10}\right|^{2}\right. \\
& \left.-2 \operatorname{Re}\left(\psi_{00} \psi_{11} \psi_{01}^{*} \psi_{10}^{*}\right)\right] .
\end{aligned}
$$

Comparing now Eqs. (5) and (7) and taking into account that $V_{\max }=6$ and $V_{\min }=4$ in the case of completely entangled and 
unentangled states of two qubits, respectively, we get

$$
C(\psi)=\sqrt{\frac{V(\psi)-V_{\min }}{V_{\max }-V_{\min }}}
$$

in the case of the general two-qubit state (4). Thus, the amount of entanglement carried by a pure two-qubit state can be determined by measurement of mean values of the basic observables given by Pauli operators (6). These observables can be directly measured in experiments, say by the SternGerlach apparatus in the case of spins, or by means of polarizers in the case of photons, etc. ${ }^{8}$

As a matter of fact, this expression (8) is equivalent to (1) for any bipartite system. ${ }^{9}$ For example, in the representation of basic observables for qutrits $(n=3)$ given in Ref. 10, the maximal and minimal values of total uncertainty in bipartite system are $V_{\max }=32 / 3$ and $V_{\min }=8$, respectively. A possible realization of qutrits is provided by biphotons. ${ }^{11}$

Equation (8) allows us to interpret concurrence (1) as a square root of the normalized total uncertainty of basic observables, specifying the system. In view of Eq. (3), the latter can be determined in terms of measurement of expectation values of the basic observables $\left\langle\psi\left|X_{\alpha}\right| \psi\right\rangle$. In other words, Eq. (8) provides an operational definition of measure of bipartite entanglement. Note also that Eq. (8) allows one to define the concurrence for any multipartite system.

Our considerations so far have applied to the pure bipartite states. In connection with mixed states, we now note that the uncertainty of an observable $X_{i}$ can be interpreted as a specific Wigner-Yanase "quantum information" about a state $\psi$ extracted from the macroscopic measurement of $X_{i}$ in this state. ${ }^{12}$ The generalization of Wigner-Yanase "information" on the case of mixed states with the density matrix $\rho$ has the form

$$
I_{i}(\rho)=-\frac{1}{2} \operatorname{Tr}\left(\left[X_{i}, \rho^{1 / 2}\right]^{2}\right) \geqslant 0
$$

It can be easily seen that in the case of pure states when $\rho$ $=|\psi\rangle\langle\psi|$ the total amount of Wigner-Yanase skew information

$$
I(\rho)=\sum_{i} I_{i}(\rho)
$$

coincides with the total uncertainty (2). The supposition is that Eq. (10) can represent a reasonable estimation from above for the amount of concurrence in the mixed bipartite state. $^{9}$

One of the authors (B.O.) would like to acknowledge the Scientific and Technical Research Council of Turkey (TÜBİTAK) for financial support.

${ }^{1} \mathrm{C} . \mathrm{H}$. Bennett and G. Brassard, in Proceedings of the IEEE International Conference on Computers, Systems, and Signal Processing (IEEE, New York, 1984), p. 175; A. Ekert, Phys. Rev. Lett. 67, 1661 (1991).

${ }^{2}$ J. Ouellette, Ind. Phys. 10, 22 (2004).

${ }^{3}$ S. Hill and W. K. Wootters, Phys. Rev. Lett. 78, 5022 (1997); P. Rungta, V. Bužek, C. M. Caves, M. Hillery, and G. J. Milburn, Phys. Rev. A 64, 042315 (2001).

${ }^{4}$ F. Minnert, M. Kuś, and A. Buchleitner, Phys. Rev. Lett. 92, 167902 (2004).

${ }^{5}$ M. A. Can, A. A. Klyachko, and A. S. Shumovsky, Phys. Rev. A 66, 02111 (2002); A. A. Klyachko and A. S. Shumovsky, J. Opt. B: Quantum Semiclassical Opt. 5, S322 (2003); A. A. Klyachko and A. S. Shumovsky, ibid. 6, S29 (2004).

${ }^{6}$ D. Kaszlikowski, D. K. L. Oi, M. Christandl, K. Chang, A. Ekert, L. C. Kwek, and C. H. Oh, Phys. Rev. A 67, 012310 (2003); T. Durt T., N. Cerf, N. Gisin, and M. Zukowski, ibid. 67, 012311 (2003); T. Durt, D. Kaszlikowski, J.-L. Chen, and L. C. Kwek, ibid. 69, 032313 (2004).

${ }^{7}$ A. Bohm, Quantum Mechanics: Foundations and Applications (Springer, New York, 1993).

${ }^{8} \mathrm{M}$. Nielsen and I. Chuang, Quantum Computation and Quantum Information (Cambridge University Press, New York, 2000).

${ }^{9}$ A. A. Klyachko, B. Öztop, and A. S. Shumovsky (unpublished).

${ }^{10}$ C. M. Caves and G. J. Milburn, Opt. Commun. 179, 439 (2000).

${ }^{11}$ A. V. Burlakov, M. V. Chechova, O. A. Karabutova, D. N. Klyshko, and S. P. Kulik, Phys. Rev. A 60, R4209 (1999); Y. I. Bogdanov, M. V. Chekhova, S. P. Kulik, G. A. Maslennikov, A. A. Zhukov, C. H. Oh, and M. K. Tey, Phys. Rev. Lett. 93, 230503 (2004).

${ }^{12}$ E. P. Wigner, Z. Phys. 131, 101 (1952); E. P. Wigner and M. M. Yanase, Proc. Natl. Acad. Sci. U.S.A. 19, 910 (1963). 